\title{
Search for the dark matter gauge bosons at the Mainz Microtron
}

\author{
H. Merkel ${ }^{* 1}$, P. Achenbach ${ }^{1}$, C. Ayerbe Gayoso ${ }^{1}$, J. Beričič ${ }^{2,3}$, J. C. Bernauer ${ }^{1, \dagger}$ \\ R. Böhm ${ }^{1}$, D. Bosnar ${ }^{4}$, L. Debenjak ${ }^{2,3}$, A. Denig ${ }^{1}$, M. O. Distler ${ }^{1}$, A. Esser ${ }^{1}$, \\ H. Fonvieille ${ }^{2}$, I. Friščić ${ }^{4}$, M. Gómez ${ }^{1}$, D. G. Middleton ${ }^{1}$, U. Müller ${ }^{1}$, M. Molitor ${ }^{1}$, \\ L. Nungesser ${ }^{1}$, J. Pochodzalla ${ }^{1}$, M. Rohrbeck ${ }^{1}$, S. Sánchez Majos ${ }^{1}$, B. S. Schlimme ${ }^{1}$, \\ M. Schoth ${ }^{1}$, F. Schulz ${ }^{1}$, C. Sfienti ${ }^{1}$, S. Širca ${ }^{2,3}$, M. Thiel $^{1}$, A. Tyukin ${ }^{1}$, A. Weber ${ }^{1}$, and \\ M. Weinriefer ${ }^{1}$ \\ ${ }^{1}$ Institut für Kernphysik, Johannes Gutenberg-Universität Mainz, D-55099 Mainz, Germany \\ 2 Jožef Stefan Institute, SI-1000 Ljubljana, Slovenia \\ ${ }^{3}$ Department of Physics, University of Ljubljana, SI-1000 Ljubljana, Slovenia \\ ${ }^{4}$ Department of Physics, University of Zagreb, HR-10002 Zagreb, Croatia \\ ${ }^{5}$ Clermont Université, Université Blaise Pascal, CNRS/IN2P3, LPC, BP 10448, F-63000 \\ Clermont-Ferrand, France \\ E-mail: merkelekph.uni-mainz.de
}

\begin{abstract}
An extra $U(1)$ boson of the dark matter sector is a possible explanation of several up to now unresolved experimental problems, e.g. the discrepency between experiment and standard model prediction for the anomalous magnetic moment of the muon. Such a boson could be detected by high intensity and high resolution fixed target experiments at existing electron facilities. In this talk the search for a $U(1)$ boson with the spectrometer setup of the A1 collaboration at the Mainz Microtron is presented.
\end{abstract}

50th International Winter Meeting on Nuclear Physics

23-27 January 2012

Bormio, Italy

\footnotetext{
* Speaker.

${ }^{\dagger}$ Present address: MIT-LNS, Cambridge, MA, USA.
} 


\section{Introduction}

The overwhelming cosmological evidence for dark matter is a very strong indication, that the current Standard Model of particle physics or our understanding of gravitation is not sufficient for a complete explanation of all existing observations. Nevertheless, the origin and nature of dark matter is up to now completely unknown. A considerable experimental effort concentrates on searches for heavy particles, motivated e.g. by supersymmetric models, either in direct production at highest energies at LHC or in indirect scattering experiments with high sensitivity.

A somewhat orthogonal approach is, not to look for the dark matter particle itself, but for a possible interaction of a dark sector. This is a quite general approach testing a large variety of possible extensions of the Standard Model, since most of these models start with a large symmetry group broken down to the known symmetries of the Standard Model. In most of these models, a remaining $U(1)$ interaction is as lowest rank local symmetry possible and sometimes even hard to avoid. E.g. during the compactification of string theories such a $U(1)$ symmetry appears in a natural way[1].

A weak interacting $U(1)$ boson is not only from the theoretical point of view interesting, but can explain a whole bunch of existing experimental puzzles. Pospelov[2] and other authors pointed out, that the existing discrepancy of the BNL measurement of the $g-2$ anomalous magnetic moment of the muon[3] with the Standard Model can be explained by an additional contribution of such a $U(1)$ boson in a loop graph (in the following we use the notation $\gamma^{\prime}$ for the $U(1)$ boson, sometimes it is called $A^{\prime}$ or $\phi$ ). Fig. 1 shows the range in mass $m_{\gamma^{\prime}}$ and mixing $\varepsilon$ between $\gamma$ and $\gamma^{\prime}$

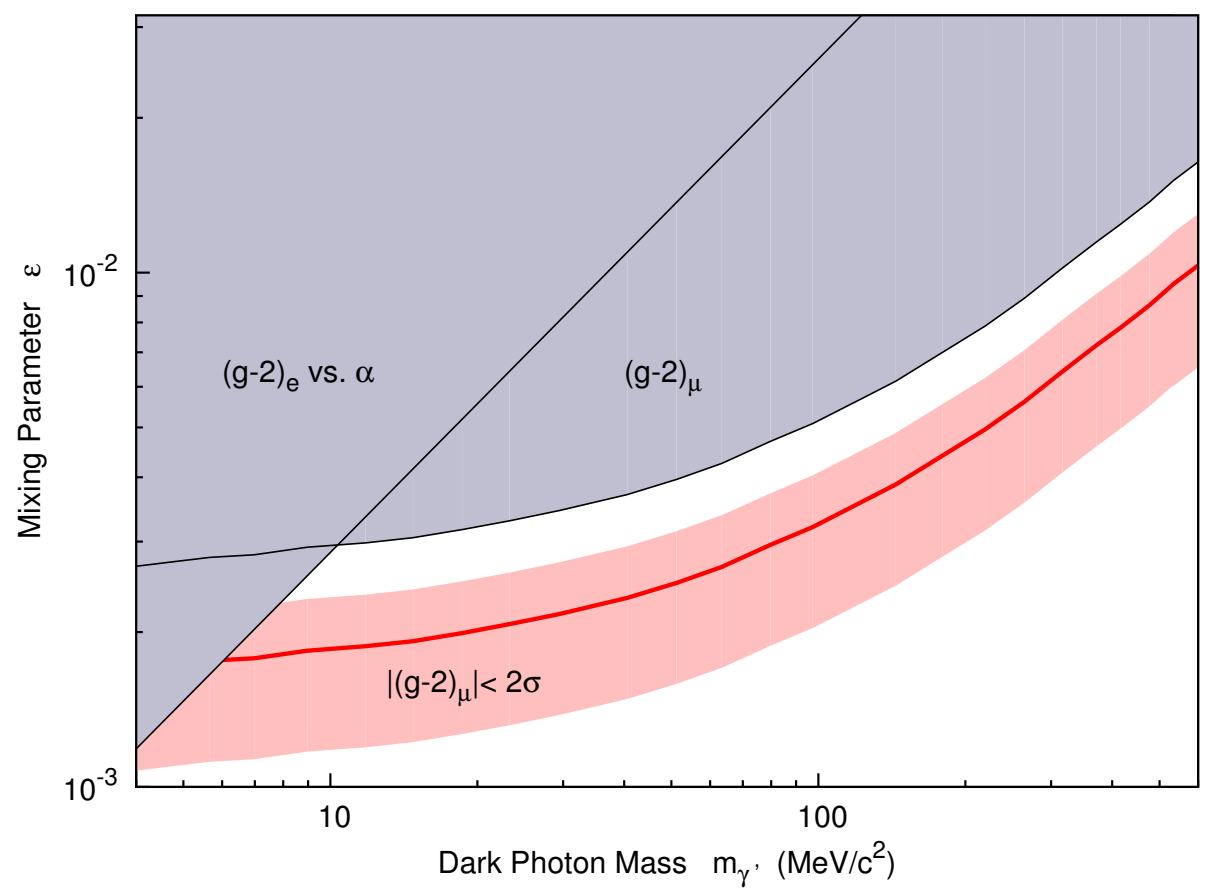

Figure 1: The range in mass $m_{\gamma^{\prime}}$ and mixing $\varepsilon$ between $\gamma$ and $\gamma^{\prime}$ to explain the experimental discrepancy (red band). The blue shaded area shows the interpretation of the experiment as exclusion limits, both for electron and muon magnetic moment. 


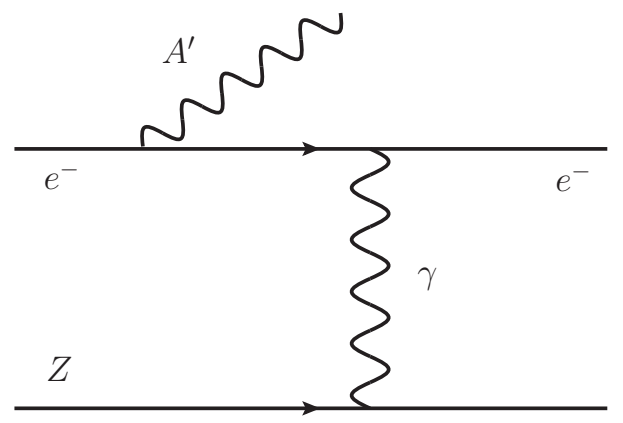

Figure 2: The mechanism of $\gamma^{\prime}$ production.

which is in accordance with the experiment.

Ref. [4] gives an overview of the experimental evidence, including several astronomical observations like e.g. the positron excess in cosmic rays, which can be explained by an additional $\gamma^{\prime}$ and which points to a mass region of this $\gamma^{\prime}$ of a few $\mathrm{MeV} / c^{2}$ up to $2 \mathrm{GeV} / c^{2}$.

A dark photon $\gamma^{\prime}$ in the expected range of coupling and mass did not show up in existing experiments due to the required high luminosity. Bjorken et al. [5] showed however, that with a dedicated high resolution fixed target experiment at a high current electron accelerator, the relevant parameter range can be tested. In this paper, a first experiment on the search of a $\gamma^{\prime}$ at the spectrometer setup of the A1 collaboration at the Mainz Microtron is presented.

\section{Electro Production of $\gamma^{\prime}$ bosons}

Figure 2 shows the production mechanism of a $\gamma^{\prime}$ in a fixed target electron scattering experiment. Since a $\gamma^{\prime}$ has the same quantum number as a real photon, the interaction of the $\gamma^{\prime}$ is governed by mixing with the photon via the kinetic term of the Lagrangian[6]. The $\gamma^{\prime}$ can therefore be produced directly by radiation off the electron, suppressed by a mixing parameter $\varepsilon$ compared to the radiation of a real or virtual photon. To increase the sensitivity, a target with high $Z$ increases the cross section by a factor of $Z^{2}$.

In Ref. [5] cross section formulas are given in the Weizsäcker-Williams approximation. Due to the small coupling, the width of the $\gamma^{\prime}$ is below any experimental resolution. After some finite flight length, the $\gamma^{\prime}$ decays into two leptons, which can be detected with high resolution magnetic spectrometers. The invariant mass of this lepton pair can be determined, and a sharp peak in this mass distribution is the unique signal of $\gamma^{\prime}$ production.

In addition to the signal, an overwhelming background of lepton pair production by radiative processes is present. Fig. 3 shows the first order radiative processes of the background (without crossed graphs). Graph (a) has the same cross section structure as the signal (only without sharp mass of the lepton pair!), while the contribution of graph (b) can be reduced by choice of the symmetric momentum distribution in the outgoing lepton pair (see fig. 4). The background contribution can be calculated exactly in QED, a first order calculation is sufficient to model the background to the percent level. 


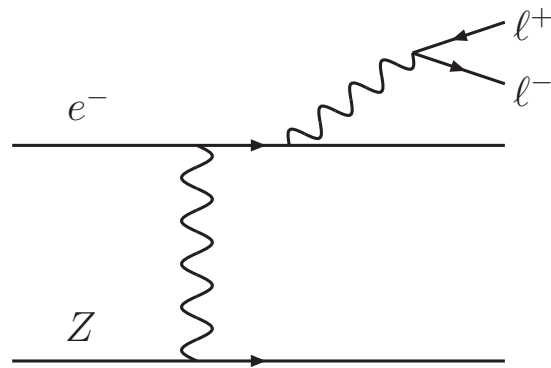

$(a)$

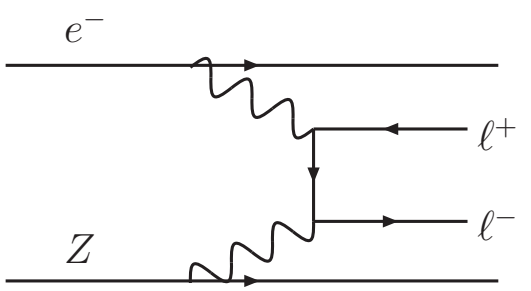

(b)

Figure 3: Background contributions by radiative processes (without crossed graphs).
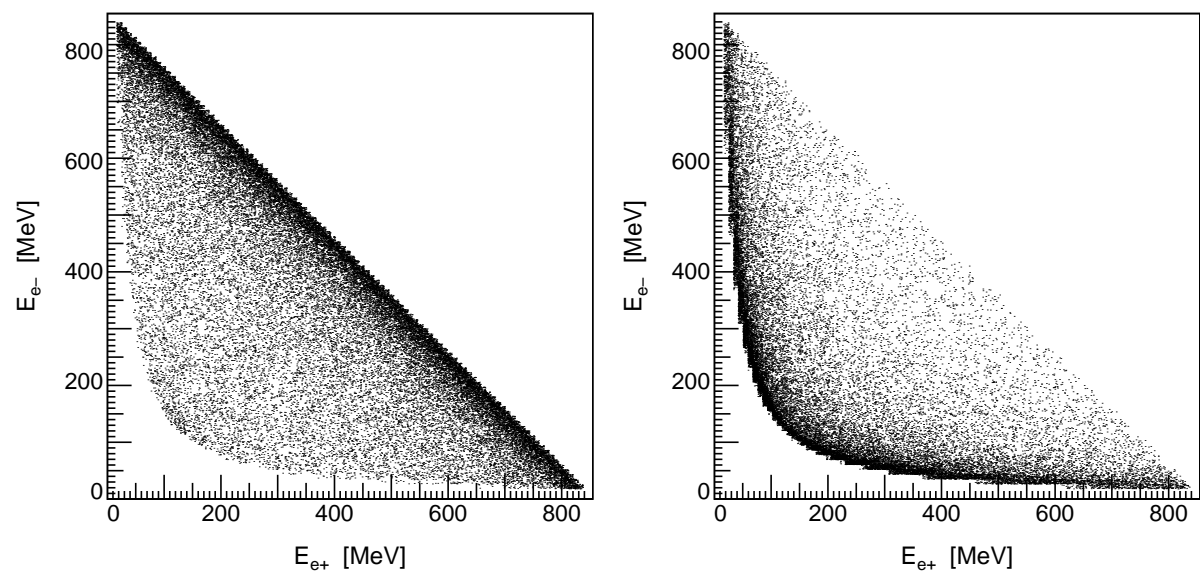

Figure 4: Bethe-Heitler contribution of graph (a) and (b) of fig. 3. While graph (a) peaks in forward direction, graph (b) peaks in the extreme asymmetric cases.

\section{Experiment}

The experiment was performed at the spectrometer setup of the A1 collaboration at MAMI (see ref. [7] for a detailed description of the setup). The MAMI accelerator can deliver a beam of up to $100 \mu \mathrm{A}$ beam current with an beam energy of up to $1600 \mathrm{MeV}$. In this first experiment, we choose an energy of $E_{0}=855 \mathrm{MeV}$ to optimize the count rate. The target consists of 12 stripes of Tantalum of $9 \mathrm{mg} / \mathrm{cm}^{2}$ thickness with a spacing of $10 \mathrm{~mm}$ and width of $2 \mathrm{~mm}$.

The scattered electron and the recoil nucleus are not detected. For the detection of the lepton pair, two high resolution spectrometers were used. Spectrometer A for the positron detection consists of a quadrupole, a sextupole and two dipoles, while spectrometer B for the electron detection consists of a single clamshell dipole. Both spectrometers are equipped with four layers of vertical drift chambers in the focal plane, two scintillator layers for timing and trigger purposes, and cherenkov detectors for the rejection of charged pions.

Table 1 summarizes the two kinematical settings used in this experiment. The settings were chosen to cover the momentum range, where the complete energy of the incoming electron is transfered to the lepton pair, emitted in forward direction. A nearly symmetric setting of the two spectrometers reduces the contribution of the QED background. 
Table 1: Kinematic settings. The incident beam energy was $E_{0}=855 \mathrm{MeV}$, and the settings are roughly centered around $E_{e^{+}}+E_{e^{-}}=E_{0}$ and $m_{\gamma^{\prime}}=250 \mathrm{MeV} / c^{2}$.

\begin{tabular}{lrccrccc}
\hline & \multicolumn{3}{c}{ Spec. A $\left(e^{+}\right)$} & \multicolumn{3}{c}{ Spec. B $\left(e^{-}\right)$} \\
& $p(\mathrm{MeV})$ & $\theta$ & $d \Omega(\mathrm{msr})$ & $p(\mathrm{MeV})$ & $\theta$ & $d \Omega(\mathrm{msr})$ & \multicolumn{1}{c}{ Events } \\
\hline Set-up 1 & 346.3 & $22.8^{\circ}$ & 21 & 507.9 & $15.2^{\circ}$ & 5.6 & $208 \times 10^{6}$ \\
Set-up 2 & 338.0 & $22.8^{\circ}$ & 21 & 469.9 & $15.2^{\circ}$ & 5.6 & $47 \times 10^{6}$ \\
\hline
\end{tabular}

Fig. 5 shows the coincidence time between the two spectrometers after cut on a lepton signal in the cherenkov detector and corrected for the flight path length in the spectrometers. A clear coincidence peak with a width of $1 \mathrm{~ns}$ FWHM can be seen. Events in a window of $\pm 1 \mathrm{~ns}$ (green shaded area) were used as identified lepton pairs. The amount of random coincidences were estimated by a cut on the sideband (red shaded area) and scaled by the width of the timing windows.

For the events in the timing window, the invariant mass of the lepton pair was calculated using the full reconstructed four momenta of the spectrometers

$$
m_{\gamma^{\prime}}^{2}=p_{e-}^{2}+p_{e+}^{2} .
$$

Fig. 6 shows the resulting missing mass distribution. The red shaded area illustrates the contribution of accidental coincidences. A $\gamma^{\prime}$ boson would show up in this spectrum as a sharp peak, with a width given by the experimental mass resolution. The expected mass resolution of less than $0.5 \mathrm{MeV} / c^{2}$ was determined by a simulation based on the measured angular and momentum resolution of the spectrometers.

\section{Results}

Based on the invariant mass distribution of fig. 6 , an exclusion limit with a confidence limit

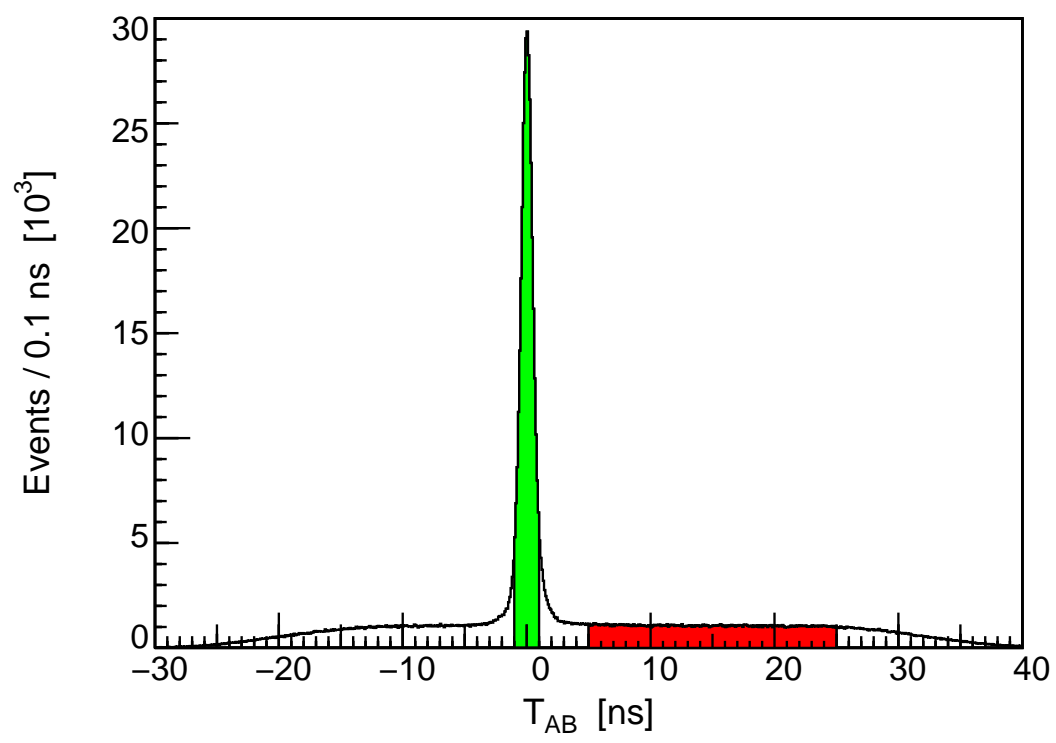

Figure 5: Coincidence time, correct for flight path length, between spectrometer A and B 


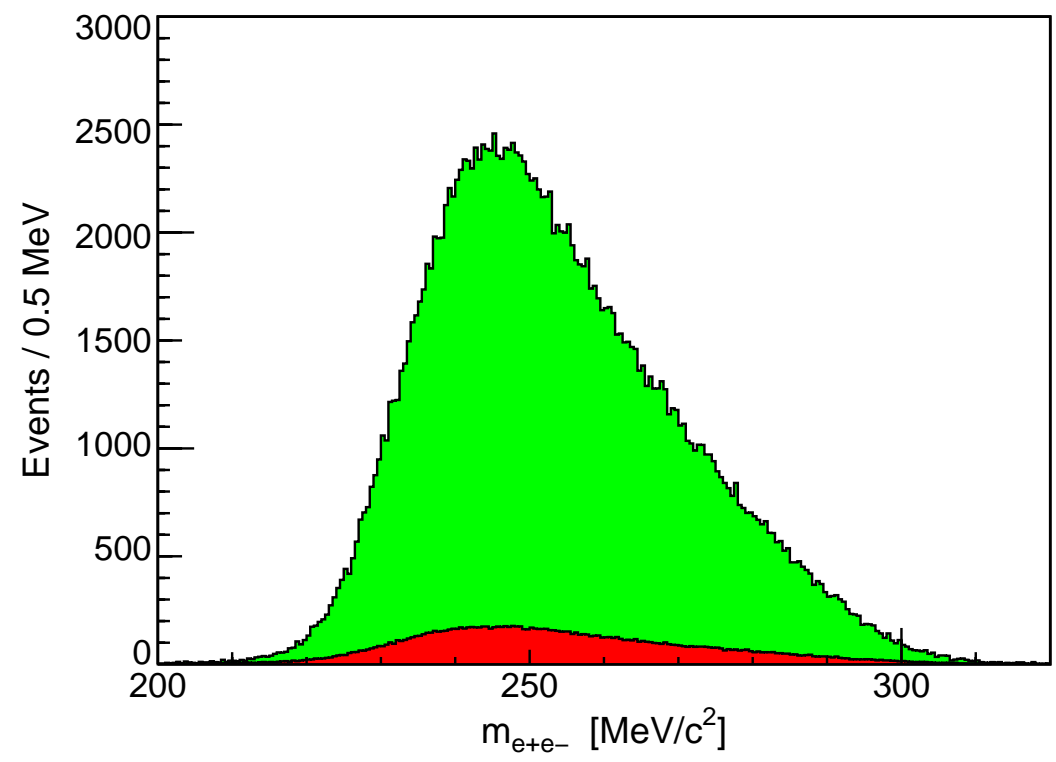

Figure 6: Invariant distribution of the electron/positron pair.

of $90 \%$ was extracted using the Feldman-Cousins algorithm[8]. As estimate for the background, the average of the neighboring bins was used. Fig 7 shows the resulting exclusion limits (blue line) after background subtraction. The red line shows the averaged exclusion limit for subjective judgement only: at a confidence limit of $90 \%$ one expects around $10 \%$ of the data points above the averaged exclusion limit.

From the exclusion limit in terms of the count rate, the exclusion limit in terms of the coupling $\alpha / \alpha^{\prime}=\varepsilon^{2}$ is calculated by normalization to the measured QED background and the background by random coincidences. Fig. 8 shows the resulting exclusion limit (averaged for the clarity of this figure only)[9] . Also drawn in this figure are the exclusion limit of the $g-2$ muon experiment and limits extracted from the BaBar data set for the decay $e^{+} e^{-} \rightarrow \gamma \mu^{+} \mu-$.

As can be seen, the existing limits on could be extended by nearly an order of magnitude in this first pilot experiment after approx. 4 days of beam time.

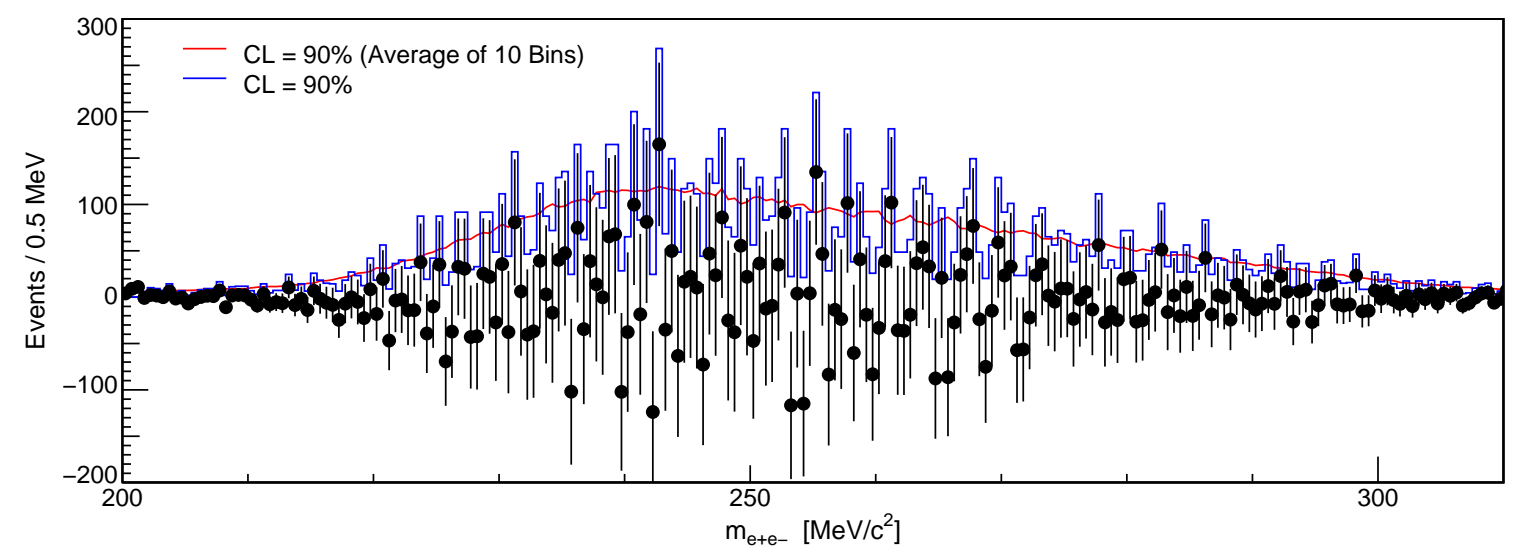

Figure 7: Exclusion limits. 


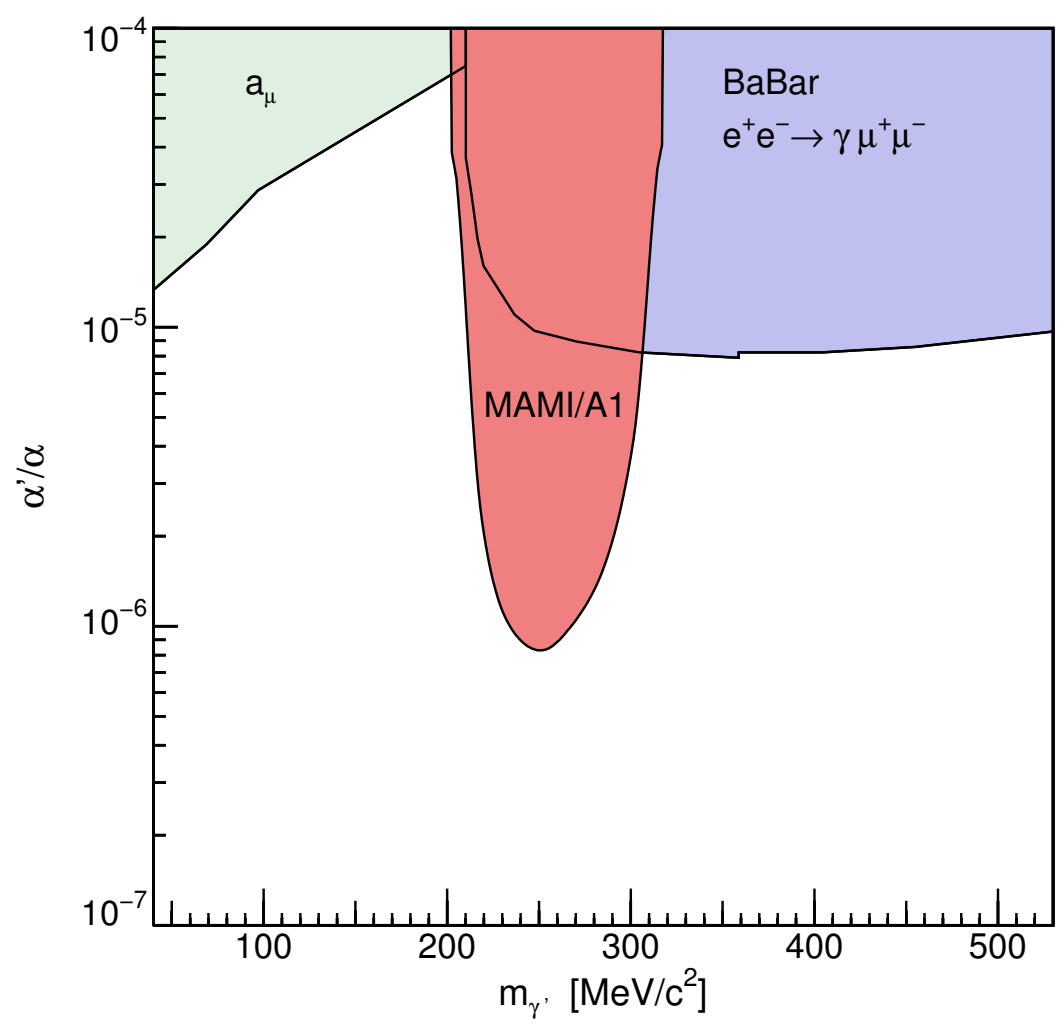

Figure 8: Exclusion limits in $\alpha / \alpha^{\prime}$

\section{Outlook}

Based on the first test experiment, a extended experimental program at several experimental facilities is currently under design. Figures 9 and 10 summarizes the parameter space of the existing proposals. Also included in this figure are areas covered by former beam dump experiments, which basically test for the reappearance of a lepton pair after a beam dump of a high energy experiment.

Just by using different beam energies without further modification of the setup, a large area of the parameter space can be excluded. These experiments have already started at A1 and are partly under analysis, a similar experimental program has started at JLab (APEX in Fig. 10).

Several proposals are made to access the region of lower $\gamma^{\prime}$ mass, which can not be reached by standard spectrometer setups due to the narrow cone of the decay leptons. The MESA project, a dedicated low energy accelerator with very high beam intensity was proposed in Mainz. A similar concept, the Dark Light project, was proposed for the JLab FEL. These experiments can cover the low mass region with sufficient overlap to the standard experiments.

At higher mass, but lower mixing strength, the luminosity of the existing accelerators is not sufficient to detect a peak on top of the QED background within a reasonable time. The lifetime of the $\gamma^{\prime}$ boson however increases and the decay length is in the region of a few micrometers up to several meters. With vertex detectors with high background rejection (HPS project at JLab) or with shielding of the production vertex (A1), the low mixing region can be reached. 


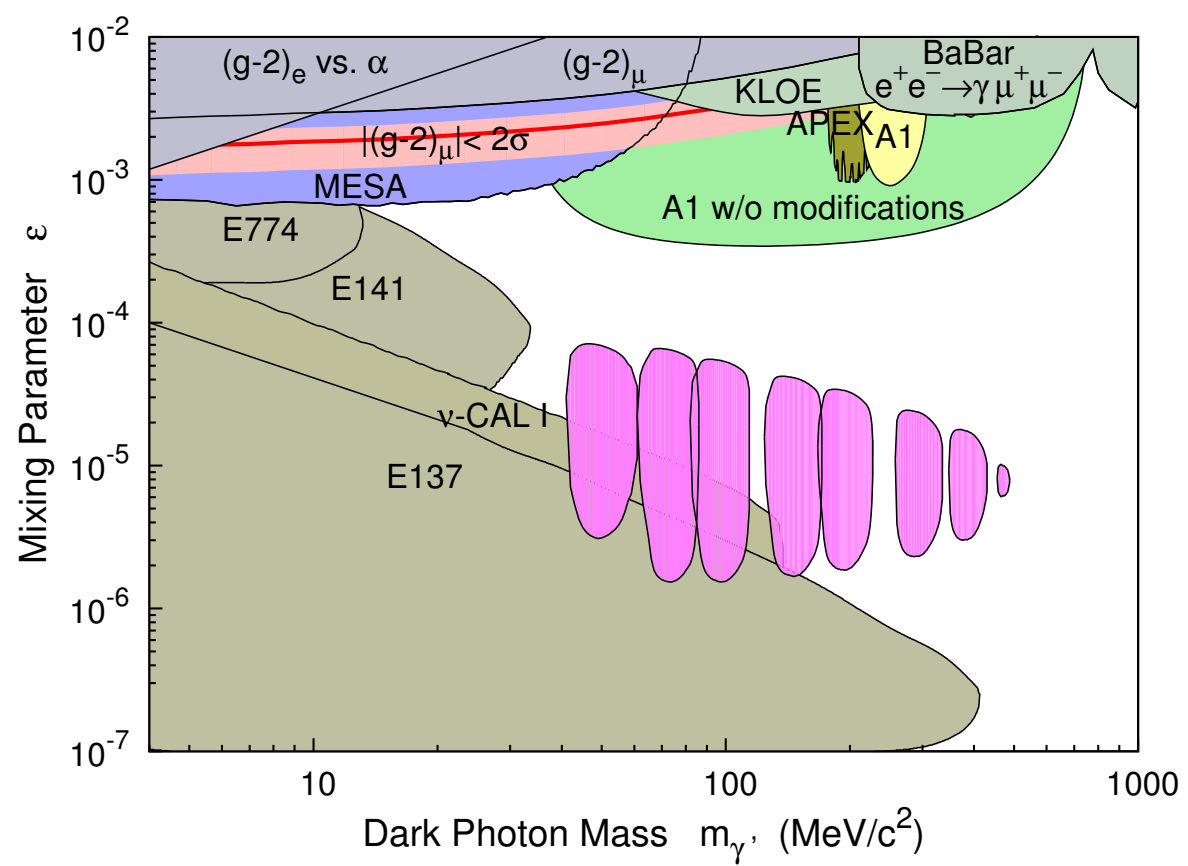

Figure 9: In this figure, the possible measurements at Mainz are marked. The green area can be covered by using the A1 setup without modifications. The blue region corresponds to the region which can be covered by the MESA project. The purple region can be accessed with shielded production vertex.

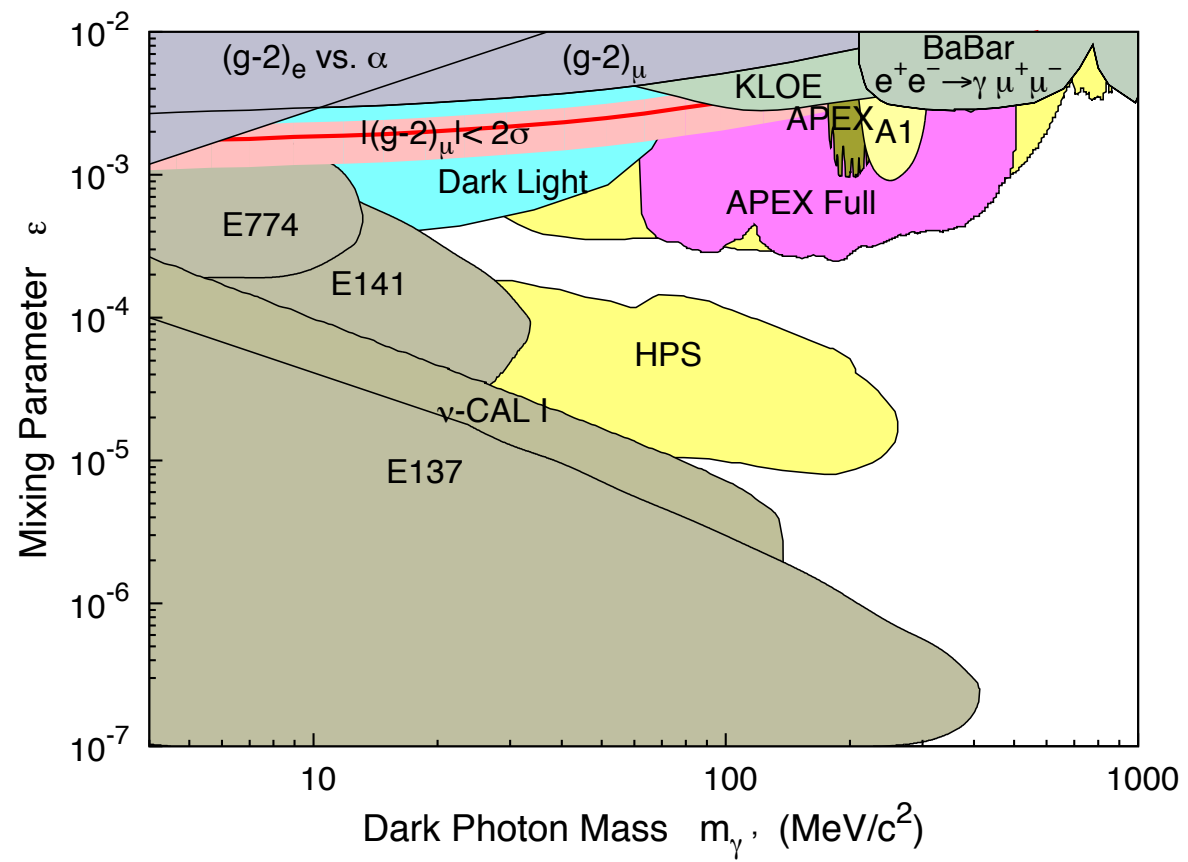

Figure 10: Other planned experiments at JLab. The purple region can be covered with the APEX experiment, the yellow region with the HPS(Heavy Photon Search) setup, and the light blue area can be covered with the Dark Light setup at the JLab FEL. 


\section{References}

[1] S. Andreas, A. Ringwald, Status of sub-GeV Hidden Particle Searches, DESY 10-137 (2010). arXiv:1008.4519.

[2] M. Pospelov, Secluded U(1) below the weak scale, Phys. Rev. D80 (2009) 095002. doi:10.1103/PhysRevD.80.095002.

[3] G. W. Bennett, et al., Final report of the e821 muon anomalous magnetic moment measurement at bnl, Phys. Rev. D 73 (7) (2006) 072003. doi:10.1103/PhysRevD.73.072003.

[4] N. Arkani-Hamed, D. P. Finkbeiner, T. R. Slatyer, N. Weiner, A Theory of Dark Matter, Phys. Rev. D79 (2009) 015014. doi:10.1103/PhysRevD.79.015014.

[5] J. D. Bjorken, R. Essig, P. Schuster, N. Toro, New Fixed-Target Experiments to Search for Dark Gauge Forces, Phys. Rev. D80 (2009) 075018. doi:10.1103/PhysRevD.80.075018.

[6] B. Holdom, Two U(1)'s and Epsilon Charge Shifts, Phys. Lett. B166 (1986) 196. doi:10.1016/0370-2693(86)91377-8.

[7] K. I. Blomqvist, et al., The three-spectrometer facility at the Mainz microtron MAMI, Nucl. Instrum. Meth. A403 (1998) 263-301. doi:10.1016/S0168-9002(97)01133-9.

[8] G. J. Feldman, R. D. Cousins, A Unified Approach to the Classical Statistical Analysis of Small Signals, Phys. Rev. D57 (1998) 3873-3889. doi:10.1103/PhysRevD.57.3873.

[9] H. Merkel, et al., Search for Light Gauge Bosons of the Dark Sector at the Mainz Microtron, Phys.Rev.Lett. 106 (2011) 251802. arXiv:1101.4091, doi:10.1103/PhysRevLett.106.251802. 